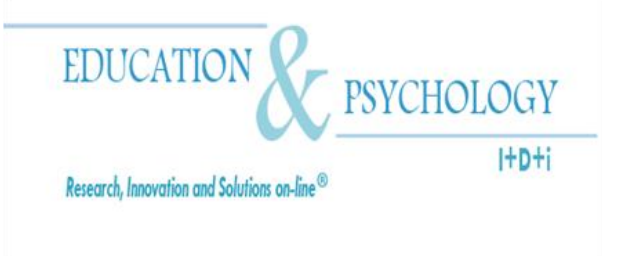

\title{
Base de Datos de Investigación en Orientación Vocacional: Enfoque Cientimétrico
}

\section{Raquel Flores Buils ${ }^{1}$, José Manuel Gil Beltrán ${ }^{1}$, Antonio Caballer Miedes ${ }^{1}$, Miguel Ángel Martínez Martínez ${ }^{1}$}

${ }^{1}$ Departamento de Psicología Evolutiva, Educativa, Social y Metodología. Universitat Jaume I, Castellón de la Plana

\section{España} Jaume I. Avd. Vicent Sos Baynat, s/n, CP. 12071, Castellón de la Plana. (España)

E-mail: flores@psi.uji.es 


\section{Resumen}

El estudio cientimétrico de la producción científica a través de las publicaciones periódicas de las Revistas especializadas no pueden llevarse a cabo exclusivamente con las bases de datos que actualmente existen. De ahí que el objetivo de este artículo sea presentar la Base de Datos en Investigación en Orientación Vocacional, basada en la utilización de Indicadores Cientimétricos.

El diseño técnico de la misma se realiza a través de tablas dinámicas, permite una actualización inmediata de los datos, así como su exportación a otros programas de cálculo, permitiendo de esta manera que el investigador pueda ampliar su campo de estudio. Todo ello, se complementa con una Clasificación Temática de Orientación Vocacional que nos permite establecer los campos objeto de mayor investigación, así como la confección de un Glosario de 245 términos de Orientación Vocacional en tres idiomas (español-inglés-francés). Por otro lado, esta Base de Datos, se puede utilizar desde una perspectiva docente como introducción a la investigación cientimétrica.

Dada la versatilidad de esta Base de Datos permite su utilización en otras áreas y campos de la Psicología.

Palabras Clave: Base de Datos de Investigación en Orientación Vocacional, Estudios Cientimétricos, Orientador, TIC. 


\title{
The Vocational Guidance Research Database: a Scientometric Approach
}

\begin{abstract}
The scientometric study of scientific output through periodical publications in specialised journals cannot be undertaken with the databases available today. Thus, this study presents the Vocational Guidance Research Database, which is based on the use of scientometric indicators.

The use of dynamic tables in the technical design of this database permits the data to be immediately updated and to be exported to other computing software programs, thus enabling researchers to expand their field of study. All this is complemented with a subject-based vocational guidance classification, which allows us to establish the most researched fields, and a vocational guidance glossary containing 245 terms in three languages (Spanish, English and French). Furthermore, we can use this database in teaching as an introduction to scientometric research and, given its versatility, it can be applied to other areas and fields of psychology.
\end{abstract}

Keywords: Vocational Guidance Research Database, scientometric studies, guidance counsellors, ICT 


\section{Introducción}

Dentro del conjunto de influencias que la ciencia de la ciencia ha recibido es importante destacar a la "Ciencia de la Documentación”, cuyos orígenes fueron el intento de control de la ingente cantidad de documentación que se empezaba a producir en las diferentes áreas del saber, allá a finales del siglo XIX, y cuyos principales responsables de su nacimiento fueron los belgas P. Otiet y H. Lafontaine. En los años sesenta, y a partir de la teoría de la recuperación de información de Vickery (1948), la ciencia de la Documentación se fue transformando en una ciencia informativa (Information Science), fundamentalmente por la influencia anglosajona, pero también alemana y soviética. La aparición de esta disciplina tuvo una gran importancia para el desarrollo de la bibliometría y de la cienciometría, herramientas metodológicas del programa de la ciencia de la ciencia (Terrada, 1983). Esta influencia ha sido mayor a partir del desarrollo, en los últimos años, de las nuevas tecnologías de la información que han permitido la creación de grandes bases de datos científicas. Especial mención merece la fundación del ISI (Institute for Science Information, Filadelfia) por Garfíeld y la publicación de los conocidos índices de referencias (Science Citation índex, Social Science Citation index).

La ciencia se configura como una organización muy compleja destinada a la actividad de producción y difusión de productos simbólicos en mercados de consumo intelectual y profesional crecientemente especializados. En estas organizaciones, la comunicación desempeña un papel fundamental. La ciencia ha de ser comunicable y accesible a todos para que pueda ser difundida y publicada por cualquiera de los cauces establecidos. Aquí, tienen un importante papel las revistas científicas.

Las revistas de este carácter aparecen en el siglo XIX. La necesidad expansiva de nuevas y cada vez más diversificadas comunidades de expertos, en pleno proceso de definición social e implantación académica, multiplicó el número de publicaciones impresas, dando lugar a la definitiva aparición, en la segunda mitad de siglo, de revistas periódicas, que pronto se convirtieron en los pilares fundamentales de las nuevas disciplinas, entre ellas la Psicología (Osier y Wozniak, 1984).

Desde una perspectiva institucional, la revista especializada es el lugar donde el trabajo científico tiene posibilidades de ser publicado, adquirir una existencia social y ser conser- 
vado. Así pues las revistas expresan el estado de la ciencia en un momento determinado, revelan los temas que preocupan, los autores o grupos más activos y amplios, y las obras de mayor influencia. En definitiva, un conjunto de datos imprescindibles para conocer la situación de una disciplina.

El nuevo contexto informático y tecnológico de nuestra sociedad ofrece condiciones innovadoras para el análisis documental. Documentalistas como Pinto, García y Agustín (2002), presentan como cambios importantes los siguientes:

1) Las bases de datos en versión impresa han dado paso a la versión electrónica.

2) La cultura acumulativa de la búsqueda documental da paso a la selectiva (documentación pertinente y filtrada en lugar de excesiva documentación).

3) Deben valorarse más los aspectos organizativos de la documentación dando paso a los procesos de reingeniería documental y al aumento de la participación del usuario en la satisfacción de sus necesidades documentales.

La llegada de los sistemas automatizados en la década de los sesenta transformó los sistemas de almacenamiento de la información. Los índices bibliográficos o revistas de resúmenes, que hasta entonces cumplían la misión de almacenar la información para su posterior recuperación, aplicaron el ordenador a las tareas de documentación con el fin de facilitar el manejo de grandes cantidades de información y empezaron a almacenar su información en soporte magnético.

Este proceso de almacenamiento y recuperación de la información ha estado estrechamente ligado al desarrollo de las tecnologías, siendo actualmente cuando se ha producido el cambio más significativo y el que ha transformado completamente la industria de la información. Esta transformación se debe principalmente a los últimos avances de la informática y de las comunicaciones que han introducido el uso del lenguaje hipertextual, que a través de enlaces permite la asociación de palabras, frases o documentos completos con información del mismo o de otros documentos, junto con la aparición de las nuevas tecnologías multimedia que permite integrar el sonido, texto, gráficos e imágenes en un único sistema de información (Tortosa y Civera, 2002).

Uno de estos avances, lo conllevan las bases de datos. Una base de datos es un conjunto de información estructurada en registros y almacenada en soporte electrónico, que puede 
facilitar la referencia de un documento, proporcionar un dato concreto o un texto al ser consultada por un ordenador o desde terminales remotos conectados a través de las redes de telecomunicaciones a grandes ordenadores, que con la ayuda de un programa informático se realiza la búsqueda y localización de información. Cada registro constituye una unidad autónoma de información que puede estar a su vez estructurada en diferentes campos (fields en inglés) o tipos de datos que se recogen en la base de datos (Tortosa y Civera, 2002).

En Orientación, cuando hablamos de base de datos, nos referimos casi exclusivamente a las grandes bases de datos que utilizamos en investigación tales como: ERIC (Educational Resources Information Center) (www.eric.ed.gov), compuesta por artículos procedentes del RIE (Resources In Education) y del CIJE (Current Index to Journals on Education); PsycINFO/PsycLIT (www.apa.org/psycinfo/about), que es sin lugar a dudas la base de datos más importante y la más consultada en el área de psicología, una base de datos bibliográfica referencial creada por PsycINFO (Psychological Abstracts Information Services) de la APA (American Psychological Association), una especie de servicios interrelacionados de información que proveen de acceso a la literatura mundial en psicología y a estudios sobre aspectos psicológicos tratados en ciencias afines; PSICODOC (psicodoc.copmadrid.org/psicodoc.htm), base de datos referencial elaborada por el Colegio Oficial de Psicólogos de Madrid con colaboración de la biblioteca de la Facultad de Psicología de la Universidad Complutense de Madrid y que recoge los trabajos publicados en revistas científicas, congresos y libros compilados procedentes de España y América Latina, cuya temática sea la psicología y disciplinas afines; las bases de datos referenciales del CSIC (Consejo Superior de Investigaciones Científicas) (www.csic.es/cbic/bases.htm), que recogen la producción científica española publicada en revistas especializadas editadas en España, como PSEDISOC, subfichero de la base de datos ISOC que recoge el área de psicología y Ciencias de la Educación.

En la actualidad, la mayoría de estas y otras bases de datos, usadas en la investigación, son accesibles bien por Internet o en CD-ROM, algunas previo pago y otras de forma gratuita (Sanz, Gil y Marzal, 2007). Sin embargo, la utilización de la base de datos como herramienta puede y debe optimizarse, sirviendo para la transmisión de aquella información que se genera en el propio proceso de enseñanza-aprendizaje. Y todo ello, de una forma lo más sencilla posible y centrándose en lo que en verdad nos interesa como profesionales de la orientación. 
La base de datos que presentamos está diseñada de acuerdo con dos premisas fundamentales y científicamente aceptadas por los investigadores:

a) El desarrollo técnico de esta base de datos se basa en la conceptualización teórica de la cienciometría. La Cienciometría, "Scientometrics", puede ser tratada también como un concepto análogo al de Bibliometría. Es una nueva área emergente de investigación. Utiliza técnicas de medición para evaluar el progreso de la Ciencia y su nivel de desarrollo, su impacto y relevancia en la sociedad. Es originaria de la antigua Unión Soviética y su primer desarrollo lo ha conocido en la Europa del Este. El término fue definido por Dobrov (1966) como "medición de los procesos informáticos", entendiendo la informática en su acepción eslava es decir "estudio de la estructura y propiedades de la información científica y las leyes de los procesos de la comunicación científica" (citados por Sengupta, 1992). Es un campo interdisciplinar no en el sentido de que se ocupe de un tema más o menos especifico ubicado entre los límites de dos disciplinas tradicionales, sino en el sentido de que involucra a un gran número de disciplinas dada la amplitud de sus objetivos.

Entre los especialistas que han intentado concretar una definición de la especialidad podemos citar a Vinckler (1991): "la Cienciometría es una disciplina científica dedicada a los aspectos cuantitativos de la Ciencia y la investigación científica". Por su parte el objetivo de la Cienciometría es, según Sengupta (1992) es evaluar cuantitativamente el crecimiento reciente de cualquier disciplina científica y los factores responsables del progreso de la actividad en esa área de conocimiento.

A pesar de que hay opiniones en otro sentido parece claro que la Cienciometría quedaría, en buena medida, englobada dentro de la Bibliometría y la Informetría. La identidad de las técnicas y metodologías es prácticamente absoluta, podríamos decir que hay un gran espacio de superposición entre la Bibliometría, la Informetría y la Cienciometría, las diferencias estribarían en que la primera tiene que ver, al menos en teoría ya que no en la práctica, con temas distintos al de la medida de la Ciencia y, por otra parte, en que ciertas medidas que interesan a la Cienciometría no derivan de las publicaciones, la financiación de la Ciencia, por ejemplo. Su talante social y su vocación de servicio a la planificación, en la que insisten distintos autores, serían otros tantos matices diferenciales. En general en esta subespecialidad el mayor énfasis se sitúa en la evaluación científica y en los procedimientos e indicadores desarrollados para conseguirla. 
Un indicador es un parámetro que se utiliza para evaluar cualquier actividad. En el caso de los indicadores cientimétricos, nos sirven para medir los avatares de la literatura científica (Araújo y Arencibia, 2002). Los aspectos a estudiar en un análisis cientimétrico son fundamentalmente cuatro, según López (1996): productividad, colaboración, materias y referencias bibliométricas.

b) Dentro de las clasificaciones que hay de las diferentes bases de datos, la base de datos que presentamos se incardina en las denominadas bases de datos documentales. Estas son sistemas de información que tratan de incorporar el mayor número de posibilidades de acceso para la localización de documentos por materias. Cada documento es sometido a un análisis documental, indización, clasificación, descriptores, identificadores, topónimos, resúmenes, etc.

Teniendo en cuenta lo expuesto anteriormente, y centrándonos en la Psicología Vocacional, el desarrollo de nuestra base de datos nos facilitará, no sólo conocer el estado de la cuestión de esta disciplina sino también su incidencia en la formación de los profesionales que se dedican al Asesoramiento Vocacional. Ello permitirá utilizar el constructivismo vocacional como un enfoque eficaz para llevar a cabo, en la actualidad, el asesoramiento de los jóvenes (Rivas, 2007).

\section{Estructura funcional de la Base de Datos de Investigación aplicada a la Orientación Vo- cacional}

La estructura orgánica de la Base de Datos de Investigación sobre Orientación Vocacional permite el acceso a la misma según sea usuario o administrador.

\section{Módulo de Administración}

Al Módulo de Administración sólo tienen acceso los miembros del presente equipo de investigación. Este bloque permite el mantenimiento y actualización de la información de la Base de Datos.

Entre las diferentes funciones que la Base de Datos nos permite llevar a cabo encontramos (ver Figura 1): 


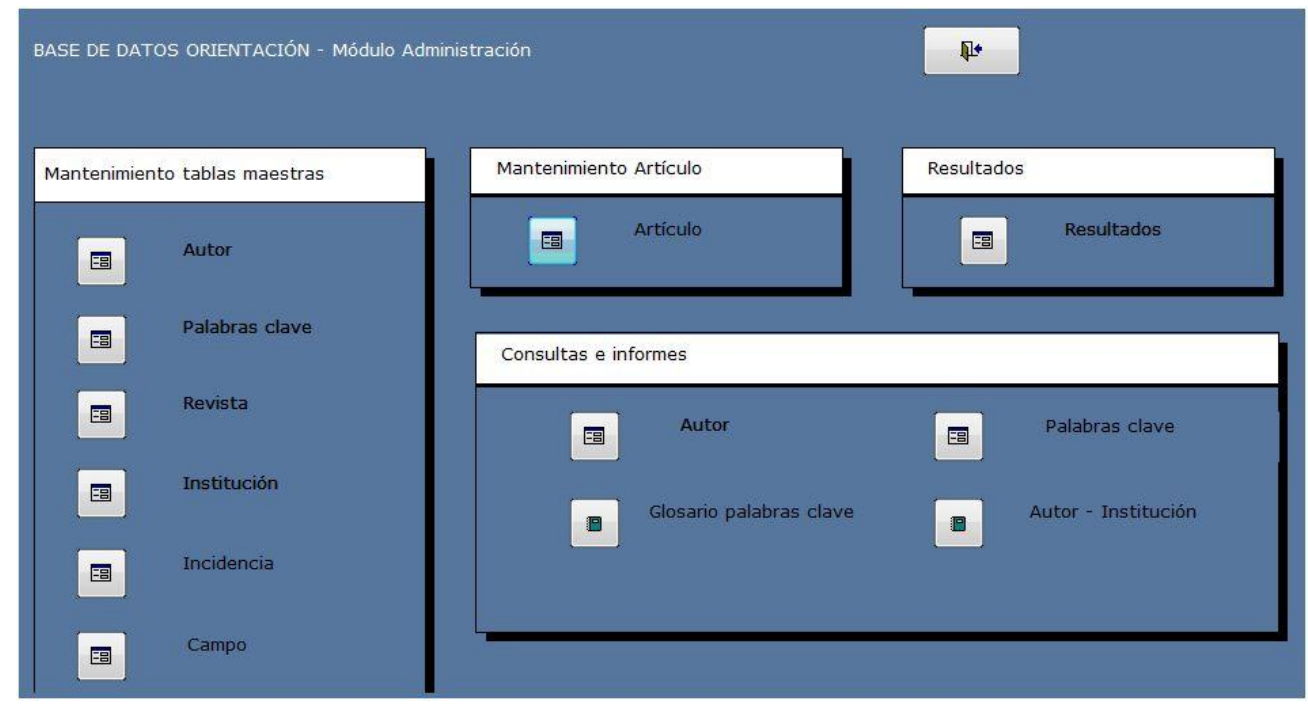

Figura 1: Módulo de Administración

a) Mantenimiento de tablas maestras

A través de este apartado tenemos acceso a los datos introducidos en la Base de Datos. De esta manera podemos tanto introducir datos nuevos como modificar los ya existentes. Estas tablas maestras son dinámicas ya que permiten la actualización inmediata de los datos ante cualquier modificación de los mismos, de manera que facilitan el cálculo automático de las distintas variables que integran esta base de datos.

Las tablas maestras de las que disponemos son:

- $\quad$ Autor: datos de los investigadores que han publicado sus trabajos en las revistas que forman parte de la Base de Datos.

- Instituciones: datos de los lugares de procedencia de los autores de los artículos: universidades, centros de investigación, etc.

- Palabras Clave: encontramos un glosario, con términos relacionados con la Orientación Vocacional. Este glosario está en tres idiomas: Español - Inglés Francés. Hay que tener en cuenta que a la hora de introducir algún nuevo término hay que realizarlo en las tres lenguas.

- Revista: nos permite poder modificar algún aspecto del nombre de las Revistas que están incluidas en la Base de Datos, así como de su descripción. También podemos incorporar revistas nuevas. 
- Incidencia: datos sobre los grupos a los que pueden dirigirse los estudios de los artículos: Orientadores, Bachillerato, etc.

- Campo: clasificación temática de Orientación Vocacional (Anexo 1)

b) Mantenimiento de los Artículos

A través de este formulario (ver Figura 2) podemos introducir nuevos artículos así como su posterior modificación.

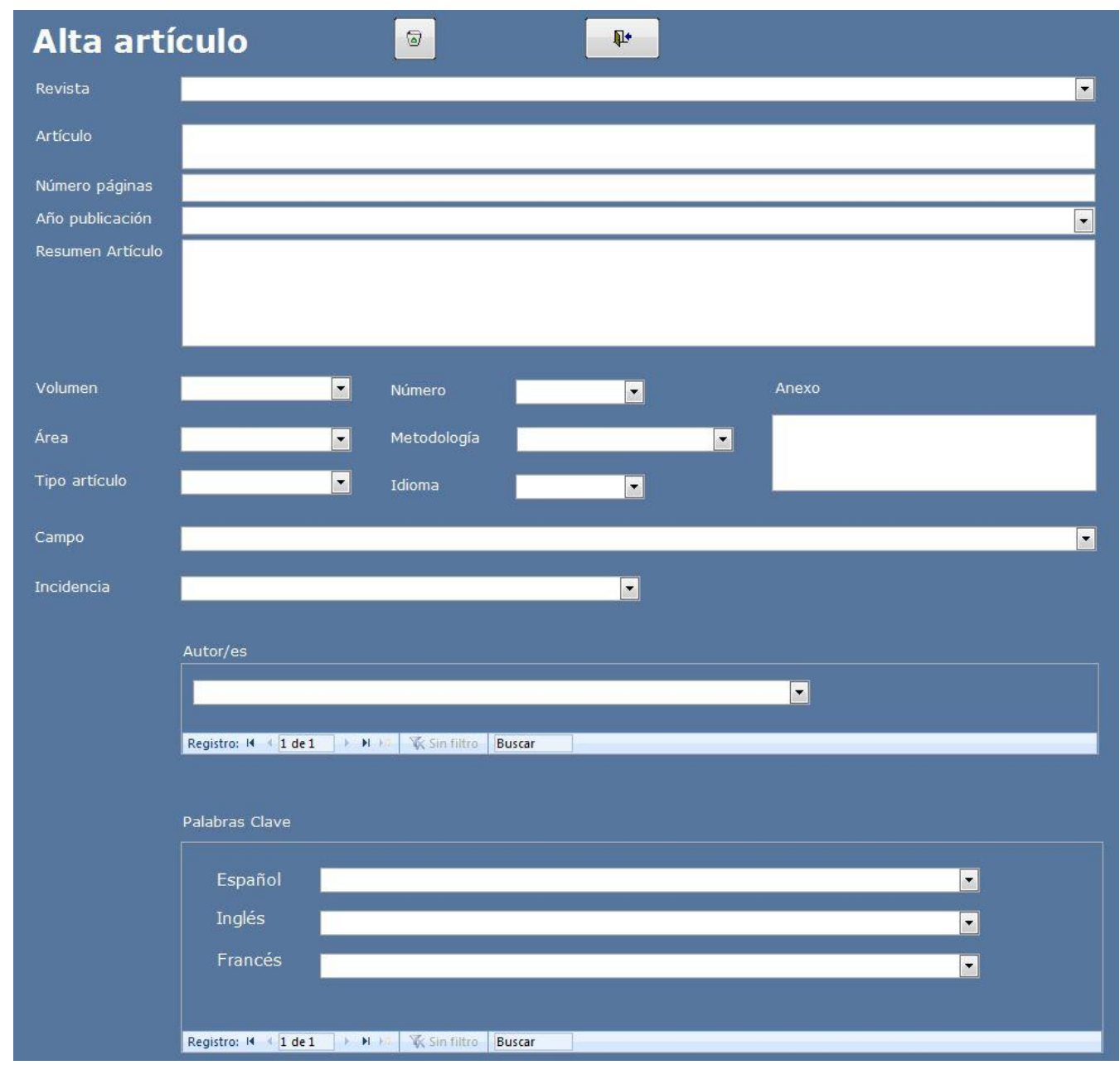

Figura 2: Formulario alta artículo

A la hora de introducir un nuevo artículo, los campos que conforman la Base de Datos y que hay que cumplimentar son los siguientes: 
- Revista

En este campo hay que indicar el nombre de la Revista a la que pertenece el artículo. Los artículos hacen referencia a la Orientación Vocacional, y abarcan el periodo 1990-2008. Las Revistas incluidas son:

- Revista Española de Orientación y Psicopedagogía (REOP)

- Bordón. Revista de Pedagogía

- Revista de Investigación Educativa (RIE)

- Revista de Psicología General y Aplicada

- L'Orientation Scolaire et Professionnelle

- Journal of Vocational Behavior

- International Journal for Educational and Vocational Guidance

En el momento en que se quiera incluir en la Base de Datos alguna otra revista, los administradores la pueden dar de alta desde este módulo.

\section{- Artículo}

De cada uno de los artículos a introducir hay que identificar los siguientes aspectos:

- Título del artículo

- Intervalo de las páginas donde se localiza el artículo

- Año de publicación

- Resumen del artículo

- Volumen y Número

- Área

En nuestro estudio hemos agrupado las áreas en tres: Orientación Vocacional, Orientación Educativa y Orientación Profesional, de acuerdo a una serie de supuestos teóricos que configuran científicamente dichas áreas (Flores, 2007). Los artículos que aparecen en la Base de Datos pertenecen en su totalidad al ámbito de la Orientación Vocacional. Para la adscripción de los artículos a esta área nos basamos en los supuestos teóricos definidos por Castaño (1983) y Rivas (2003). Por otro lado, la herramienta está preparada para incluir artículos que pertenezcan a las áreas mencionadas anteriormente. Así mismo, también se contempla la posibilidad de introducir nuevas áreas de estudio. 


\section{- Metodología}

Siguiendo la nomenclatura científica al uso, hemos clasificado cada uno de los artículos en los siguientes tipos: teórico, práctico y empírico. El artículo clasificado como teórico es aquel que desarrolla conceptos, teorías, historia, desde un punto de vista conceptual y de paradigma. Incluimos como artículo práctico las experiencias y/o aplicaciones que se derivan del texto analizado, incluyendo a veces anexos que le dan esa vertiente aplicada. Finalmente, clasificamos los artículos en el tipo empírico, cuando estos siguen las normas del proceso científico: hipótesis, objetivos, procedimiento, muestra, conclusiones.

Cada uno de los trabajos empíricos ha sido analizado y clasificado según el tipo de diseño utilizado en el estudio siguiendo la siguiente clasificación (adaptada de Reig, 1989):

1. Metodología Experimental y Cuasiexperimental

a) Diseños experimentales de comparación de grupos

b) Diseños de caso único

c) Diseños cuasiexperimentales

d) Otros

2. Metodología de Encuestas

a) Diseños de encuesta transversal

b) Diseños de encuesta longitudinal

c) Diseños de encuesta descriptiva

d) Diseños de encuesta explicativa o analítica

e) Diseños ex post facto

f) Otros

3. Metodología Observacional

4. Metodología Cualitativa

a) Entrevista en profundidad

b) Grupo de discusión

c) Otros

\section{Otros}
a) Metaanálisis
b) Bibliometría
c) Otros 


\section{- Idioma}

Identifica el idioma en que está escrito el artículo (español, inglés, francés, italiano, alemán, etc.).

\section{- Incidencia}

Esta variable nos indica el ámbito donde el artículo tiene su mayor grado de aplicación. Para ello hemos establecido dos parámetros. El primero abarca distintas etapas educativas del Sistema Educativo Español: Educación Secundaria, Bachillerato y Universidad. El segundo, hace referencia a ámbitos más extensos y con temática más variada, tales como: adolescencia, adultos, familia, mundo laboral, orientadores y profesorado. Así mismo incluimos la categoría de Todos, cuando la incidencia abarca a muchos de estos ámbitos. Estos ámbitos pueden ser ampliados teniendo en cuenta las nuevas situaciones sociales, educativas, etc., que influyen en la orientación vocacional. De manera que cuando se quiera trabajar con otras áreas el ámbito de incidencia puede variar, de ahí la flexibilidad que ofrece esta base de datos para adecuarse a la realidad.

\section{- Campo}

Viene definido en su acepción más amplia como el Análisis de Contenido. Así, una clasificación por temas o áreas de los artículos tratados en una revista durante un tiempo determinado, nos da una idea acerca de los intereses teóricos que han predominado dentro de una comunidad científica en dicho periodo (Tortosa y Civera, 2002).

Aquí incluimos la Clasificación Temática de Orientación Vocacional (Flores, 2010) (ver Anexo 1). Esta clasificación es una actualización de la propuesta de Adame (2000), la cual también puede ser modificada con el fin de dar respuesta a los nuevos aspectos que la literatura científica nos pueda presentar.

\section{- Anexo}

Este apartado lo consideramos de gran interés ya que en ocasiones el artículo presenta anexos con información muy válida tanto para el investigador como para el profesional de la Orientación. Así puede incluir cuestionarios, guías, escalas, etc. Esto evita que información interesante pueda perderse con el paso del tiempo. 


\section{- Autores}

En la literatura bibliométrica, una de las escalas tradicionales utilizadas como medida de eminencia científica es el número de trabajos publicados por cada autor en revistas científicas conocidas (Carpintero y Peiró, 1983; Prpic, 1996; Adair y Vohra, 2003). Así pues, resulta de interés conocer y profundizar en la distribución de trabajos de los científicos de acuerdo con el tamaño de su producción escrita. Desde el análisis de la productividad, medida a través del número de publicaciones de la revista, las publicaciones de los autores, de un grupo de investigación, de las instituciones, en un país, o en un determinado número de tiempo, obtenemos el grado de crecimiento de la ciencia (Smith et al, 1998).

La Base de Datos ofrece un desplegable con todos los autores que han publicado alguno de los trabajos que forman parte de ella. Estos autores están vinculados a la Institución a la que pertenecen (universidades, institutos, organizaciones, etc.). En el caso de que se vaya a incluir un autor que no esté registrado, la misma Base de Datos lo detecta y envía directamente al administrador a la Tablas Maestras de autores. Tras incluir los datos del nuevo autor, pasarán a formar parte de la misma.

\section{- Palabras Clave}

Los sistemas de recuperación de la información, para una práctica eficaz, requieren de unos dispositivos o símbolos que permiten identificar el contenido principal del documento, destacándolo del conjunto de la colección sin perder tiempo por parte del usuario. A este proceso de aislar en cada documento los trazos descriptivos se le llama indización. Así la UNESCO (1975) la define desde un doble punto de vista: como proceso, consiste en describir y caracterizar un documento con la ayuda de representaciones de los conceptos contenidos en dicho documento; en cuanto a su finalidad, va destinada a permitir una búsqueda eficaz de las informaciones contenidas en un fondo documental. La indización es un requisito documental no sólo para un adecuado almacenamiento, sino también para la oportuna recuperación de la información.

Al respecto, hemos elaborado un Glosario de términos de Psicología y Orientación Vocacional. Para su realización, se consultó el Tesauro Europeo de los Sistemas Educativos (Comisión Europea, 2009), el Glosario de términos de Orientación de la AIOSP/ONISEP (2001) (Association internationale d'orientation scolaire et professionnelle-Office National d'Information Sur les Enseignements et les Professions) y el Glosario de Orientación escolar y profe- 
sional de la Universidad de Santiago de Compostela (Sobrado y Porto, 2000). Tras su consulta, fueron seleccionados aquellos términos relacionados con el área vocacional. A estos términos, se incorporaron aquellas palabras claves correspondientes a los artículos de las revistas que hemos analizado.

El glosario se compone de 245 términos que se presentan en tres idiomas "EspañolInglés-Francés", de esta manera se facilita la búsqueda de artículos en cualquiera de estos idiomas. Al igual que sucede en otros campos, en el caso de que se vaya a incluir una palabra clave nueva, la misma Base de Datos lo detecta y envía directamente al administrador a la Tablas Maestras de palabras clave. Tras incluir los nuevos datos, pasarán a formar parte de la misma.

\section{- Bibliografía}

Las referencias bibliográficas aportadas por los autores de los artículos científicos, nos dan abundante información sobre el uso en las diversas comunidades científicas. Vemos, por ejemplo, que los científicos sociales realizan referencias bibliográficas de más libros que los científicos pertenecientes a las disciplinas duras, que utilizan en mayor proporción artículos científicos. Podemos ver también el ritmo de envejecimiento de la literatura científica según la vida media de los trabajos referenciados, que varía también en función del campo estudiado.

El análisis de referencias bibliográficas indica qué autores, trabajos, temáticas, etc., son más relevantes. Así, analizaremos por cada artículo el autor, los autores referenciados, el tipo de referencia (libro, capítulo de libro o revista), año de la referencia y el idioma (ver Figura 3).

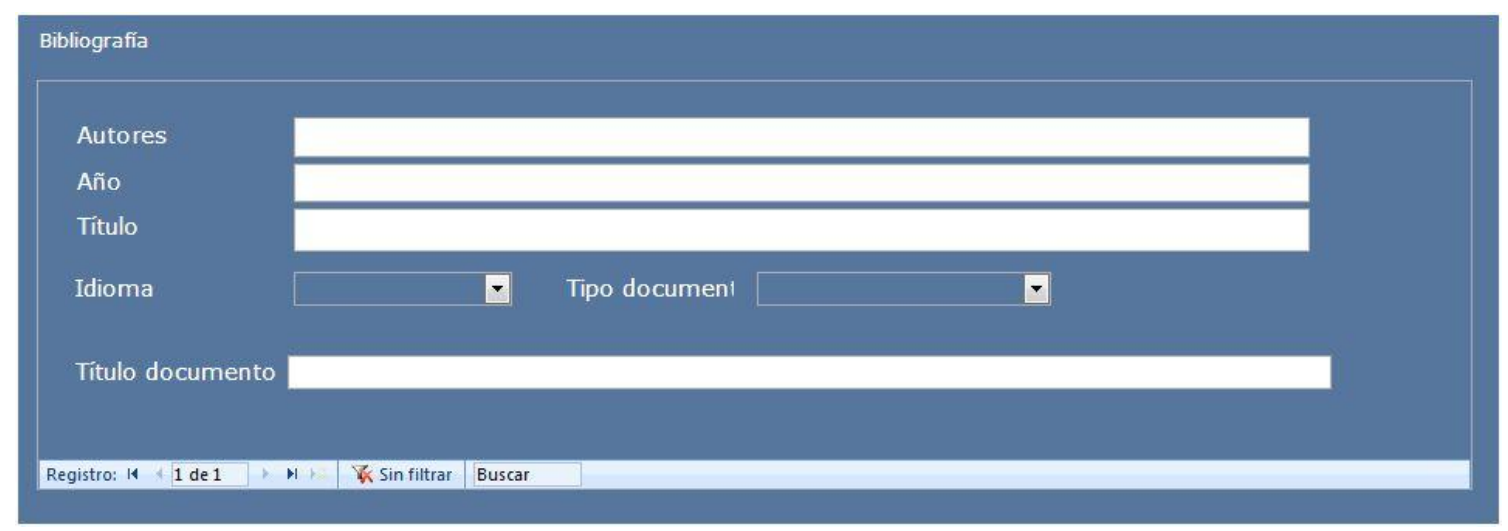

Figura 3: Formulario alta bibliografía artículo 
Los administradores, para cada referencia señalarán:

- Autor/es de referencia

- Año de la referencia

- Idioma de la referencia

- Título (a cumplimentar en el caso de artículos de revistas o de capítulos de libros)

- Tipo de documento (libro, revista, capítulo de libro, comunicación, conferencia, tesis doctoral, trabajo de investigación, documento on-line, test, congreso, otros documentos)

- Título del documento

\section{c) Consultas e informes}

Los administradores también pueden realizar desde su módulo consultas o búsquedas de artículos. Estas consultas se pueden llevar a efecto por: autor, palabra clave y/o realizar una consulta general en la que se filtrará la información por cualquier campo.

Es posible hacer estas búsquedas a través de las relaciones previamente establecidas. Éstas quedan definidas en la Figura 4.

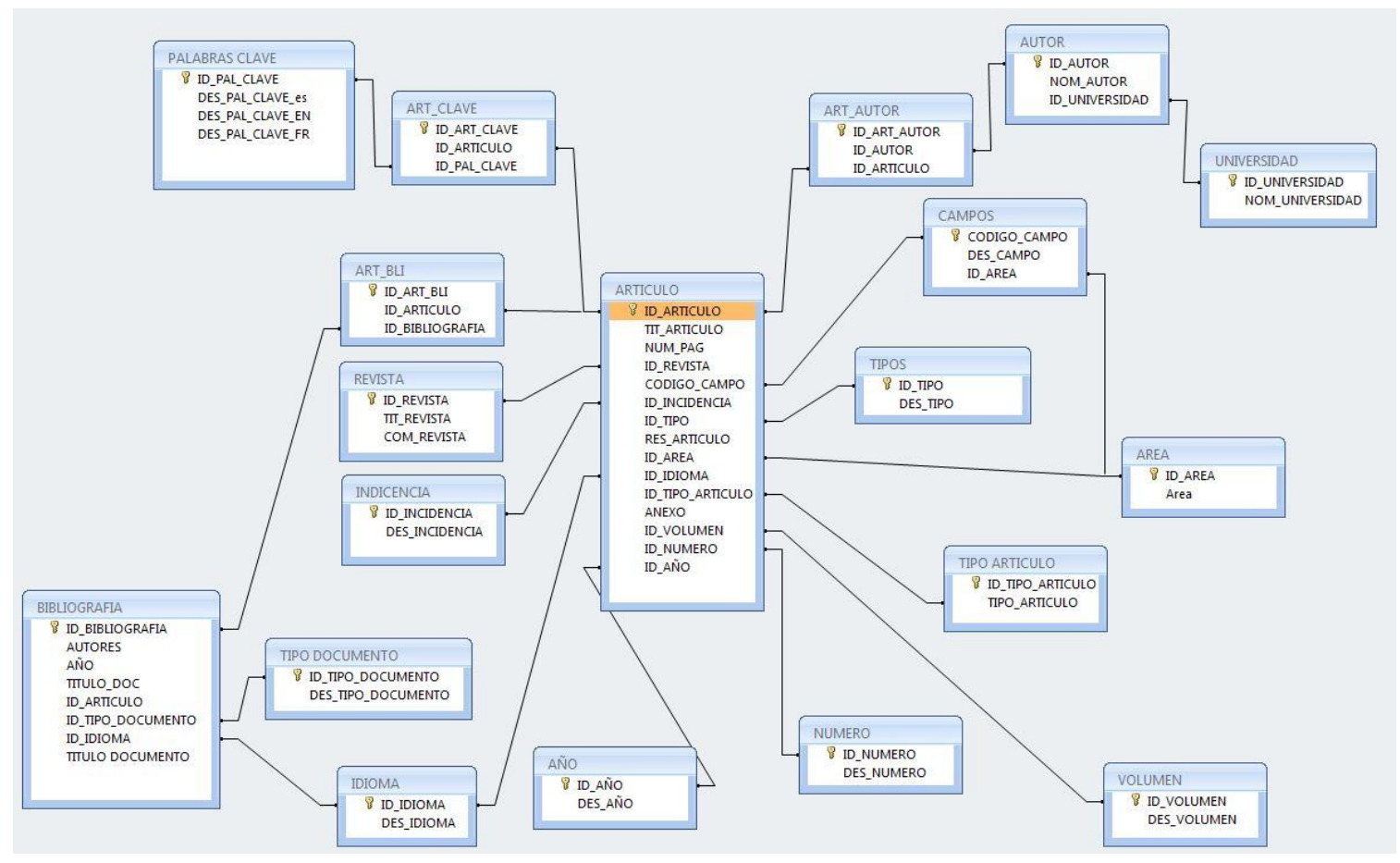

Figura 4: Relaciones entre los campos de la base de datos 


\section{- Consulta por autor}

Esta consulta nos permite obtener todos los artículos que aparecen en la Base de Datos que han sido escritos por algún autor en concreto. Para facilitar la búsqueda, aparece un glosario con el nombre de todos los autores y la institución a la que pertenece.

De cada uno de los artículos que surgen de la búsqueda obtenemos: información sobre la institución a la que pertenece el autor; título del artículo; revista en la que está publicado; datos de identificación (número, volumen, año, páginas); idioma en el que está escrito; el resumen del artículo; metodología que sigue; tipo de artículo; campo al que pertenece; a quien va dirigido; si posee algún anexo (material) relevante; en el caso de que estuviera escrito por más de un autor; los nombres de los autores colaboradores; y las palabras claves que identifican el artículo.

\section{- Consulta por palabra clave}

Permite poder realizar una búsqueda de aquellos artículos que tengan asignada una palabra clave en concreto. Es muy importante señalar que como estas palabras están introducidas en español, francés e inglés, al realizar la búsqueda de los artículos que correspondan a una palabra clave en un idioma también obtendremos aquellos artículos que posean esta palabra en cualquiera de los otros dos idiomas.

Hay que señalar que para facilitar la realización de este tipo de consultas, se ha habilitado en el apartado de consultas e informes, un glosario con todas las palabras clave que aparecen en los artículos incluidos en la base de datos. De cada artículo que obtengamos al realizar la consulta, nos aparecerá la información de los campos que hemos comentado en la Consulta por Autor.

\section{- Consulta general}

Con este tipo de consulta se pueden localizar los artículos que nos interesen en base a cualquiera de los campos que aparecen en el formulario, e incluso se pueden ir filtrando datos, conjugando varios campos para acotar los resultados de la búsqueda. Para ello, partimos del formulario alta-artículo (Figura 2), donde vamos seleccionando aquellos campos que nos interesan. De cada artículo que obtengamos al realizar la consulta, nos aparecerá la información de los campos que hemos comentado en los tipos de consulta anteriores (ver Figura 5). 


\begin{tabular}{|c|c|c|c|c|}
\hline TIT_REVISTA & $\begin{array}{l}\text { indicencia } \\
\text { DES_INCIDENCIA }\end{array}$ & CODIGO_CAMPO & DES_CAMPO & TIT_ARTICULO \\
\hline $\begin{array}{l}\text { Revista de Investigación Educativa } \\
\text { (RIE) }\end{array}$ & Orientadores & A.1. & Origen y desarrollo histórico & $\begin{array}{l}\text { Roles y funciones de los } \\
\text { orientadores }\end{array}$ \\
\hline Journal of Vocational Behavior & Orientadores & A.1. & Origen y desarrollo histórico & $\begin{array}{l}\text { Vocational Psychology and Career } \\
\text { Counseling: Inventing the Future }\end{array}$ \\
\hline Journal of Vocational Behavior & Orientadores & A.1. & Origen y desarrollo histórico & $\begin{array}{l}\text { Adapting Vocational Psychology to } \\
\text { Cope with Change }\end{array}$ \\
\hline Journal of Vocational Behavior & Orientadores & A.1. & Origen y desarrollo histórico & $\begin{array}{l}\text { The Developmental Perspective in } \\
\text { Vocational Psychology }\end{array}$ \\
\hline Journal of Vocational Behavior & Orientadores & A.1. & Origen y desarrollo histórico & $\begin{array}{l}\text { John L. Holland's Contributions to } \\
\text { Vocational Psychology: A Review } \\
\text { and Evaluation }\end{array}$ \\
\hline $\begin{array}{l}\text { Revista de Investigación Educativa } \\
\text { (RIE) }\end{array}$ & Orientadores & A.1. & Origen y desarrollo histórico & Y, ċlos servicios sirven? \\
\hline Journal of Vocational Behavior & Orientadores & A.1. & Origen y desarrollo histórico & $\begin{array}{l}\text { Dynamic Forces in the Growth and } \\
\text { Change of Vocational Psychology }\end{array}$ \\
\hline $\begin{array}{l}\text { Revista de Investigación Educativa } \\
\text { (RIE) }\end{array}$ & Universidad & A.1. & Origen y desarrollo histórico & $\begin{array}{l}\text { Oferta de los servicios de } \\
\text { orientación en las universidades } \\
\text { españolas. }\end{array}$ \\
\hline $\begin{array}{l}\text { International Journal for } \\
\text { Educational and Vocational } \\
\text { Guidance }\end{array}$ & Orientadores & A.1. & Origen y desarrollo histórico & $\begin{array}{l}\text { An analytical framework for career } \\
\text { research in the post-modern era }\end{array}$ \\
\hline
\end{tabular}

Figura 5: Ejemplo de informe

\section{d) Resultados}

En este apartado se nos permite cuantificar los datos de las distintas variables que conforman la base de datos (ver Figura 6).

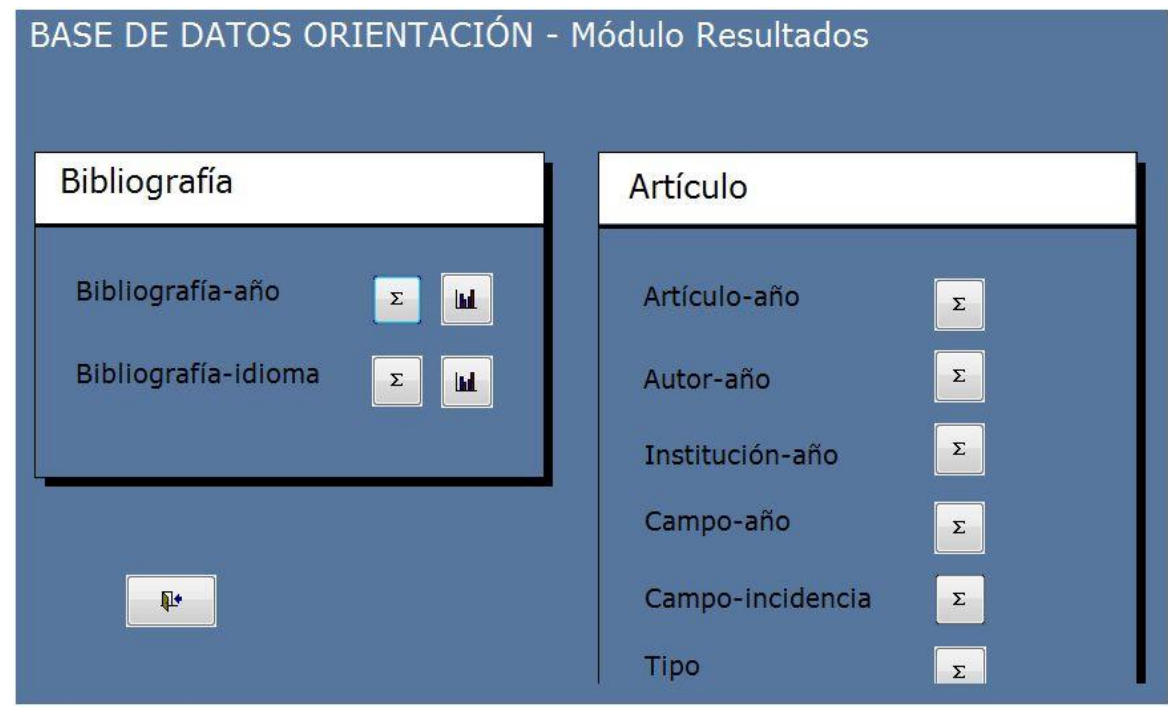

Figura 6: Módulo resultados

- Respecto a los artículos, podemos obtener resultados sobre:

- Artículo-Año: podemos obtener cuántos artículos sobre orientación vocacional se han publicado por año en cada una de las revistas.

- Autor-Año: nos permite saber el número de autores que han publicado sus trabajos por año en cada una de las revistas. 
- Institución-Año: nos muestra por años, qué instituciones son las más productivas en cada una de las revistas.

- Campo-Año: podemos comprobar por años cuáles han sido las temáticas más trabajadas por los autores en cada una de las revistas

- Campo-Incidencia: nos muestra en cada revista, el número de trabajos publicados según la temática tratada en cada artículo y según al grupo de referencia al que se dirige.

- Tipo: podemos encontrar el número de trabajos teóricos, empíricos y prácticos que se han publicado en cada una de las revistas.

- Respecto a las referencias bibliográficas de los artículos, podemos obtener los siguientes resultados:

- Bibliografía-Año: nos permite conocer por años, el número de referencias bibliográficas que los autores han utilizado. Estos datos podemos verlos representados en una gráfica donde también podemos seleccionar los años o las revistas que nos interesen.

- Bibliografía-Idioma: nos ofrece información sobre el tipo de documento que se ha utilizado en las referencias y el idioma en que está dicho documento. Estos datos también los podemos obtener representados en una gráfica dinámica donde podemos seleccionar aquellos datos que queremos que nos aparezcan.

La base de datos nos permite crear informes de acuerdo a las variables seleccionadas. Al mismo tiempo nos ofrece la posibilidad de exportar los datos a un Microsoft Excel, con lo que el investigador amplía su campo de trabajo en el tratamiento de los mismos.

\section{Módulo de Usuario}

A este Módulo pueden acceder todas aquellas personas interesadas en obtener la información que la Base de Datos posee. Entre las diferentes funciones que el usuario puede realizar, encontramos (ver Figura 7). 


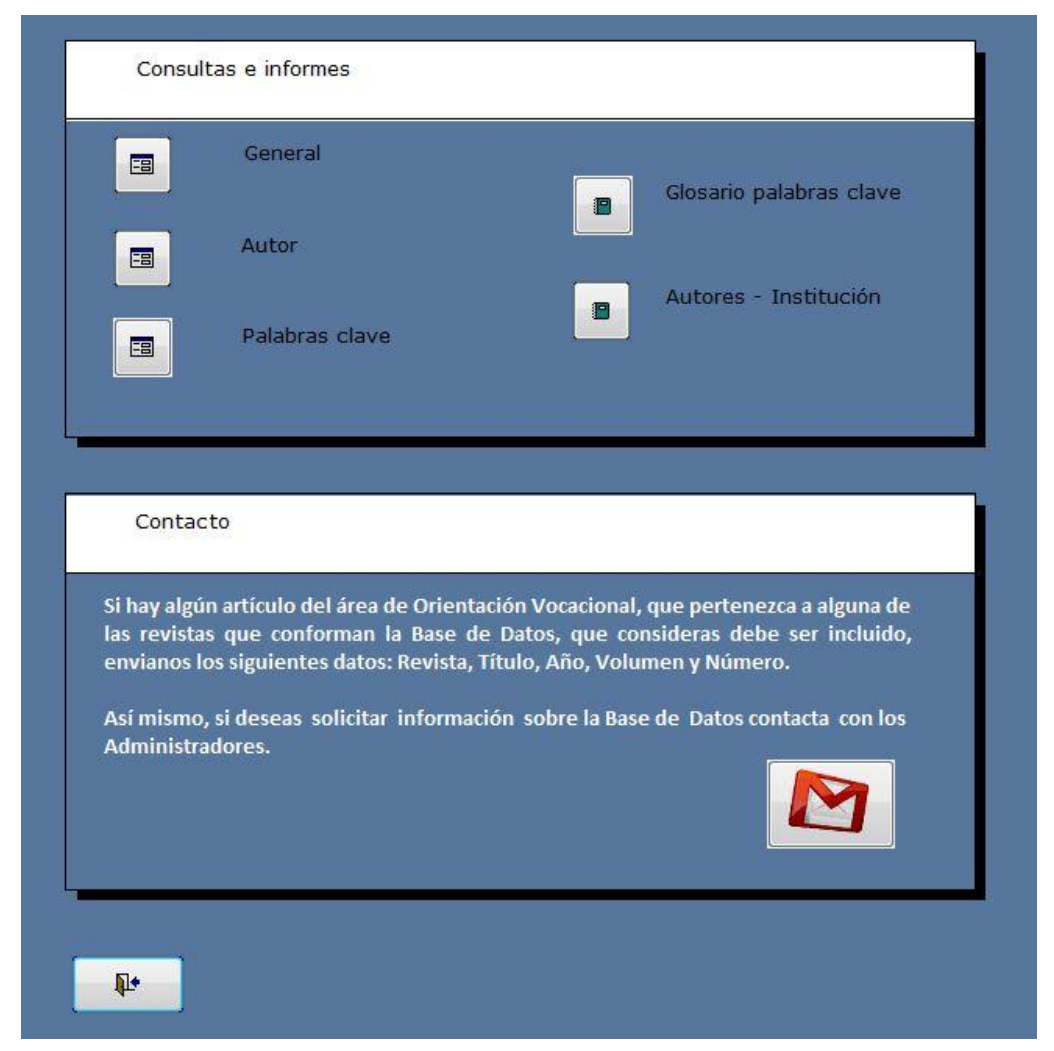

Figura 7: Módulo usuario

a) Consultas e informes

Los usuarios pueden realizar desde este módulo consultas o búsquedas de artículos. Estas consultas se pueden llevar a cabo por: autor, palabra clave y/o realizar una consulta general. Al igual que en el módulo de los administradores, los usuarios pueden obtener un informe con los datos de su consulta. La estructura y las funciones de este bloque, son las mismas que las que hemos explicado en el módulo de administración.

\section{b) Contacto}

Los usuarios a través de este apartado pueden solicitar consultas a los administradores. Pueden realizar dos tipos de consultas diferentes, por un lado pueden enviarnos los datos de algún artículo de orientación vocacional que pertenezca a alguna de las revistas que forman parte de la base de datos, si consideran que debería ser incluido.

Para ello harán llegar desde la misma base de datos, los datos de identificación del artículo, estos los recibirán los administradores, quienes valoraran si ha de ser incluido o no. Si se considera que debe ser incluido, los administradores son los encargados de introducirlo en la base de datos, controlando así los artículos que son incorporados. Por otro lado, si algún 
usuario quiere algún tipo de información sobre la base de datos, así como obtener algún resultado de los datos, puede solicitarlo a los administradores.

\section{Conclusiones}

La Base de Datos de Investigación en Orientación Vocacional constituye una aportación importante al estudio de la psicología y orientación vocacional desde la perspectiva cientimétrica, ya que nos permite conocer de manera rápida y actual cuál es la situación de esta área a través de las publicaciones científicas que se realizan en las revistas. Así, el objetivo del presente artículo ha quedado cubierto, al presentar una base de datos que permite al investigador realizar estudios de corte cientimétrico, aspecto que la diferencia de las bases de datos al uso (psicodoc, eric, psicinfo, scopus, etc.), ya que éstas suelen utilizarse como "buscadores de información". Las aportaciones técnicas que se incluyen en nuestra base de datos hacen referencia fundamentalmente a: uso de tablas dinámicas, realización de informes, posibilidad de impresión, así como transferir los datos a otros programas de cálculo como el excel. además de lo descrito anteriormente, el carácter innovador de la citada base de datos radica en su utilización: por investigadores, profesionales de la orientación vocacional y profesorado y estudiantes universitarios para sus prácticas de investigación en las materias relacionadas con la psicología vocacional.

Las aportaciones de la Base de Datos, las podemos clasificar en los siguientes apartados:

A) Respecto a la Psicología y Orientación Vocacional nos permite:

- Llevar a cabo estudios de indicadores cientimétricos, ya que las bases de datos que existen actualmente no permiten realizar directamente estudios de este tipo.

- Nos ofrece un conocimiento real y actual sobre el estado de cuestión de la Orientación Vocacional, lo cual nos permitirá establecer una línea base para poder desarrollar programas de intervención para la formación de los Orientadores del siglo XXI y promover nuevas líneas de investigación adecuadas a las necesidades actuales de la sociedad.

- Facilitar a los Orientadores y profesionales de este ámbito un material que les pueda ser útil en su labor de evaluación, diagnóstico, intervención y/o asesoramiento, acercándoles trabajos y experiencias concretas de interés, sistematizando esta información y facilitando su acceso. 
- Ofrece a los Investigadores una recopilación de documentación relevante sobre Orientación Vocacional, convirtiéndose en un material de gran utilidad a la hora de buscar y seleccionar información para la realización de cualquier tipo de investigación.

- Realizar búsquedas de artículos a través de una palabra clave en un idioma, que automáticamente lleva a la búsqueda de este término en los otros dos idiomas que conforman el glosario.

- El glosario de Orientación Vocacional que incluye esta base de datos abarca tanto la búsqueda de artículos como el aspecto formativo de los investigadores, al unificar la terminología científica de la Orientación Vocacional.

B) Respecto a su aplicación generalizada, podemos afirmar que esta Base de Datos puede convertirse en una base de datos para la investigación en Orientación Educativa y Orientación Profesional, ya que los parámetros específicos de estas dos áreas han sido ya establecidos (Flores, 2007). Por otro lado, la configuración técnica de esta Base de Datos permite adaptarla con facilidad al estudio de otras áreas de la Psicología: clínica, social, etc.

C) La configuración técnica de la Base de Datos hace que pueda ser utilizada con facilidad tanto para los investigadores como para cualquier usuario.

- Permite exportar datos a programas estadísticos como el Microsoft Excel, facilitando el uso y manejo de los datos.

- Sirve como material práctico para la formación de los estudiantes universitarios, tanto para completar su formación como para iniciarles en la investigación en este campo.

- Será accesible a través de la Web del orientador europeo, por lo que generará un acercamiento entre la investigación y la práctica profesional.

- La posibilidad de sacar informes con los campos seleccionados, así como guardar y/o imprimirlos, constituye una utilidad práctica de dicha Base de Datos.

- El hecho de que en su configuración se contemplen tanto las tablas como los gráficos dinámicos, hace posible su actualización inmediata.

Por otro lado, el acceso a la misma, será gratuito y se integrará como un elemento más de la estructura del portal Web del Orientador Europeo (en construcción). En definitiva creemos que la utilización de nuevas tecnologías puede facilitar el dar respuestas eficaces a los retos que a los orientadores les plantea la sociedad del siglo XXI. En el caso concreto de esta Base de Datos, queremos señalar que una aportación importante a nivel general es que nos ofrece la 
posibilidad de establecer una comunicación directa entre los investigadores y los profesionales de la Orientación Vocacional, de modo que la ciencia llegue a todos.

\section{Referencias}

Adair, J. G. y Vohra, N. (2003). The explosion of knowledge, references, and citations: Psychology s unique response to a crisis. American Psychologists, 58(1), 15-23.

Adame, M. T. (2000). Orientación y Psicología Vocacional en España: Revisión y Estado de la cuestión (1970-1999). Tesis doctoral (no publicada). Universitat de les Illes Balears.

AIOSP/ONISEP (2001). Glosario de términos de orientación. Association internationale d'orientation scolaire et professionnelle (AIOSP) and Office National d'Information Sur les Enseignements et les Professions (ONISEP).

Araújo, J. A. y Arencibia, R. (2002). Informetría, bibliometría y cienciometría: aspectos teórico-prácticos. ACIMED, 10 (4), 165-170.

Carpintero, H. y Peiró, J. M. (1983). The significance of the bibliometric methodology to the studies of the history of psyhcology. Revista de Historia de la Psicología, 4(1), 21-32.

Castaño, C. (1983). Psicología y Orientación Vocacional. Madrid: Morata.

Comisión Europea (2009). Tesauro Europeo de los Sistemas Educativos. Extraído el 1 de abril de 2010 de http://www.educacion.es/cide/jsp/plantilla.jsp?id=eurydice032009

Dobrov, G.M. (1966). Nauka o Nauke. Kiev: Naukova

Flores, R. (2007). La Orientación en España. Una aproximación a través de la Revista Española de Orientación y Psicopedagogía (1990-2006). Trabajo de investigación no publicado, Universitat Jaume I.

Flores, R. (2010). Psicología y Orientación Vocacional. Estudio Cientimétrico. Un reto en la Formación del Orientador Europeo del siglo XXI. Tesis doctoral no publicada, Universitat Jaume I.

López, P. (1996). Introducción a la Bibliometría. Valencia: Promolibro

Osier, D. y Wozniak, R. (1984). A century of serial publications in psychology 1850-1950. New York: Kraus International.

Pinto, M.a.; García, F.J. y Agustín, M. ${ }^{a}$ del C. (2002). Indización y resumen de documentos digitales y multimedia. Técnicas y procedimientos. Gijón (Asturias): TREA

Prpic, K. (1996). Characteristics and determinants of eminent scientists' productivity. Scientometrics, 36(2), 185-206.

Reig, F. (1989). Psicología Escolar, Psicología Vocacional y Orientación Escolar a través de las Revistas Bordón, Revista Española de Pedagogía y Revista de Psicología General 
y Aplicada. (1970-1985). Tesis de licenciatura no publicada, Universitat de València. Valencia.

Rivas, F. (2003). Asesoramiento Vocacional. Teoría, práctica e instrumentación. Barcelona: Ariel.

Rivas, F. (2007). ¿Conducta y asesoramiento vocacional en el mundo de hoy? Electronic Journal of research in Educational Psychology, 5 (1), 5-14.

Sanz, J., Gil, J.M. y Marzal, A. (2007). Herramientas Informáticas para la Orientación y el Asesoramiento Vocacional. Electronic Journal of research in Educational Psychology, $5(1), 201-232$.

Sengupta, I. N. (1992). Bibliometrics, informetrics, scientometrics and librametrics; an overview. Libri, 42 (2), 75-98.

Smith, M. C.; Locke, S. G.; Boisse, S. J., Gallagher, P. A.; Krengel, L. E.; Kuczel, J. E.; Rapoo, B. y Wertheim, C. (1998). Productiviry of educational psychologists in Educational Psychology Journal 1991-1996. Contemporary Educational Psychology, 23(2), 173-181.

Sobrado, L. y Porto, A. (2000). Glosario de Orientación escolar y profesional de la Universidad de Santiago de Compostela. Servicio de Publicaciones e Intercambio Científico de la Universidad de Santiago de Compostela, en colaboración con la Asociación Internacional para a Orientación Escolar e Profesional. Extraído el 12 de febrero de 2010 de http://www.usc.es/snl/term/Vocabularios/orient-escolar.pdf

Terrada, M.L. (1983). La documentación médica como disciplina. Valencia. Centro de Documentación e Información Biomédica de la Universidad de Valencia.

Tortosa, F. y Civera, C. (2002). Nuevas tecnologías de la información y documentación en Psicología. Barcelona: Ariel.

UNESCO (1975). Principes d'indexation. París: UNESCO

Vickery, B. C. (1948). Bradford's law of scattering. Journal of Documentation, 4 (1), 198203.

Vinkler, P (1991). Possible causes of differences in information impact of journals from different subfields. Scientometrics, 20 (2), 145-161. 


\section{Anexo 1. Clasificación Temática de Orientación Vocacional}

\section{A. PSICOLOGÍA VOCACIONAL / ORIENTACIÓN VOCACIONAL}

A.1. Origen y desarrollo histórico

A.2. Conducta vocacional / vocación: concepto, teorías vocacionales

A.3. Asesoramiento vocacional / orientación vocacional

A.3.1. Enfoques del asesoramiento / orientación vocacional: fundamentos teóricos-tecnológicos

A.3.2. Diagnóstico / evaluación vocacional

A.3.3. Programas / sistemas de intervención y asesoramiento / orientación de la conducta vocacional

A.3.4. Asesor, equipos y servicios de orientación vocacional

A.3.4.1. Formación de Orientadores

A.3.5. Asesoramiento / orientación vocacional por grupos de edad/etapas educativas o formativas

A.3.6. Asesoramiento / orientación vocacional en el curriculum escolar

A.4. Desarrollo vocacional / desarrollo de la carrera

A.4.1. Autoconcepto / autorrealización

A.4.2. Aptitudes / capacidades para el desarrollo vocacional

A.4.3. Intereses / preferencias vocacionales

A.4.4. Toma de decisiones vocacionales

A.4.5. Indecisión vocacional

A.4.6. Elección vocacional

A.4.7. Madurez vocacional

A.4.8. Congruencia-ajuste vocacionales

A.4.9. Conducta vocacional y género

A.4.10. Desarrollo vocacional de colectivos minoritarios

A.4.10.1. Minorías étnicas

A.4.10.2. Minorías funcionales: deficiencias físicas, psíquicas y sensoriales

A.4.10.3. Grupos y/o colectivos de inadaptación social

A.5. Información Vocacional 
A.6. Investigación en Psicología / Orientación Vocacional

A.6.1. Instrumentación

A.6.2. Metodología de investigación

A.6.3. Fuentes, medios.

A.7. Asesoramiento / orientación vocacional por países, comunidades / situación comparativa.
A.7.1. Políticas de Orientación

A.8. TIC

A.8.1. Sistemas de Asesoramiento Computerizados

A.8.2. Internet

A.9. Cultura y Multiculturalidad

A.10. Otros 